\title{
Emergence of NDM-producing Pseudomonas aeruginosa among hospitalized patients and impact on antimicrobial therapy during the coronavirus disease 2019 (COVID-19) pandemic
}

\author{
Leandro Reus Rodrigues Perez $\mathrm{PhD}^{1,2}$ (1), Eliana Carniel BSc ${ }^{3}$ and Gabriel Azambuja Narvaez MD \\ ${ }^{1}$ Federal University of Health Sciences of Porto Alegre, Porto Alegre, Rio Grande do Sul, Brazil, ${ }^{2}$ Qualitá Laboratory, Novo Hamburgo, Rio Grande do Sul, Brazil, \\ ${ }^{3}$ Feevale University, Novo Hamburgo, Rio Grande do Sul, Brazil and ${ }^{4}$ Mãe de Deus Hospital, Porto Alegre, Rio Grande do Sul, Brazil
}

To the Editor-Pseudomonas aeruginosa is a leading cause of nosocomial infection, mainly in intensive care patients. Notably, $P$. aeruginosa has a wide variety of resistance mechanisms that often restrict therapeutic options. Although carbapenems are considered potent agents for the treatment of Pseudomonas infections, the increased prevalence of carbapenem resistance among them has been observed worldwide in past decade. ${ }^{1}$

Distinct mechanisms, such as OprD porin loss, overexpression of MexAB-OprM efflux pump, and carbapenemase production may confer a carbapenem-resistance phenotype in $P$. aeruginosa. ${ }^{1,2}$ Among the carbapenemase enzymes, metallo- $\beta$-lactamases (MBLs) have one of the most potent resistance mechanisms because they inactivate virtually all $\beta$-lactam agents, except aztreonam. Different types of MBLs have been described among $P$. aeruginosa isolates, particularly the SPM-1 type in Brazil, with varied degrees of endemicity. ${ }^{3,4}$ Nowadays, other MBLs have emerged around the world, mainly New Delhi metallo- $\beta$-lactamases (NDMs), which are present largely in Enterobacterales, but also in nonfermenters (Acinetobacter and Pseudomonas spp). ${ }^{5}$

The current antimicrobial pipeline offers short-term treatment of infections due to MBL producers. Thus, our most important tool against the spread of MBL producers relies on intensified infection control, surveillance, and antimicrobial stewardship policies. We sought to determine the prevalence of carbapenem-resistance and MBL production and to assess the impact of them on resistance rates to other antimicrobial agents among $P$. aeruginosa nosocomial isolates.

From October 2020 to March 2021, P. aeruginosa isolates were recovered from hospitalized patients in Porto Alegre city and its metropolitan area as part of a survey study on antimicrobial resistance. Only the first isolate of each patient was included in the survey. Isolates were confirmed as $P$. aeruginosa based on oxidase and pyocyanin production or, for atypical isolates, by MALDITOF (bioMérieux, Marcy l'Etoile, France). Susceptibility was determined using the disk diffusion for amikacin, cefepime, ceftazidime, ciprofloxacin, imipenem, meropenem and piperacillintazobactam, and the results were interpreted according to CLSI guidelines. ${ }^{6}$ Polymyxin B susceptibility was determined using broth microdilution. All isolates resistant to carbapenems were

Author for correspondence: Leandro Reus Rodrigues Perez, E-mail: leandro.reus@ gmail.com

Cite this article: Perez LRR, Carniel E, and Narvaez GA. (2022). Emergence of NDMproducing Pseudomonas aeruginosa among hospitalized patients and impact on antimicrobial therapy during the coronavirus disease 2019 (COVID-19) pandemic. Infection Control \& Hospital Epidemiology, 43: 1279-1280, https://doi.org/10.1017/ ice. 2021.253 subjected to the NG-test CARBA 5 multiplex immunochromatographic assay for the detection of $b l a_{\mathrm{KPC}}, b l a_{\mathrm{OXA}-48-\mathrm{like}}, b l a_{\mathrm{NDM}}$, $b l a_{\mathrm{VIM}}$, and $b l a_{\mathrm{IMP}}$ carbapenemases (NG Biotech, Gulpry, France) and were further confirmed by polymerase chain reaction assay (PCR). When an MBL enzyme was detected, an in vitro synergism between ceftazidime/avibactam and aztreonam was evaluated using a disk-diffusion approximation test, as published elsewhere, with modifications.

Statistical analyses were carried out using SPSS version 13.0 software (IBM, Chicago, IL). Prevalence ratio (PR) and 95\% confidence intervals (CIs) were calculated. $P$ values were calculated using the $\chi^{2}$ or Fisher exact test.

In total, $278 P$. aeruginosa isolates were recovered from 156 patients during the study period. Among the 156 nonduplicate isolates, $83(53.2 \%)$ were recovered from intensive care patients. These were more prevalent in respiratory secretions $(n=88$; $56.4 \%)$, urine $(\mathrm{n}=34 ; 21.8 \%)$, blood $(\mathrm{n}=16 ; 10.3 \%)$, and other secretions $(\mathrm{n}=18 ; 11.5 \%)$.

Overall, higher resistance rates were noted for meropenem (47.4\%), followed by imipenem (43.6\%), cefepime (39.1\%), piperacillin/tazobactam (37.2\%), ciprofloxacin (33.3\%), ceftazidime (32.7\%), amikacin (28.2\%), and gentamicin (22.4\%).

An MBL-producing $P$. aeruginosa isolate was recovered at least once from 27 (17.3\%; 95\% CI, 12.2\%-24.0\%) of these 156 patients, and the $b l a_{\mathrm{NDM}-1}$ was the sole MBL enzyme detected. All isolates were susceptible to polymyxin $B$. However, NDM-1 production was responsible for a high $\beta$-lactam resistance rate (Fig. 1). Additionally, resistances to amikacin $(74.1 \%$ vs $17.0 \%$; PR, $4.34 ; 95 \%$ CI, $2.1 \%-9.0 \%$; $P<.001)$, gentamicin $(70.4 \%$ vs $12.4 \%$; PR, $5.67 ; 95 \% \mathrm{CI}, 2.6 \%-12.4 \%$; $P<.001)$, and ciprofloxacin (100.0\% vs $19.4 \%$; PR, $5.16 ; 95 \%$ CI, $2.6 \%-10.2 \% ; P<.001)$ were significantly higher among NDM-1 producers than nonproducing $P$. aeruginosa isolates.

Table 1 illustrates the susceptibility profile of the 27 NDM1 -producing $P$. aeruginosa isolates. Ceftazidime/avibactam plus aztreonam showed an in vitro synergistic result in all NDM isolates, despite the resistance to both drugs when they were individually tested.

The relatively low prevalence of carbapenem-resistance driven by MBL enzyme revealed in this survey allowed us to discourage coverage with empirical therapy because there are therapeutic options for non-carbapenemase-producing $P$. aeruginosa isolates (eg, ceftolozane/tazobactam, data not shown). However, we emphasize the potential for dissemination of an NDM-1-producing $P$. aeruginosa among critically ill patients in Southern Brazil which possibly could displace other MBL enzyme (mostly SPM-1) or another resistance mechanism as driver for carbapenem resistance. 
Table 1. Susceptibility Profile, PMB Minimum Inhibitory Concentration (MIC) and Ceftazidime/Avibactam Plus Aztreonam Synergism Among 27 NDM-Producing P. aeruginosa Isolates

\begin{tabular}{lllr}
\hline No. (\%) of Isolates & Susceptibility To ${ }^{a}$ & PMB MIC $_{90}$ & No. (\%) of CZA plus ATM Synergism + \\
\hline $15(55.5)$ & PMB & $1.0 \mu \mathrm{g} / \mathrm{mL}$ & $15(100)$ \\
\hline $7(25.9)$ & PMB, AMK, and GEN & $1.0 \mu \mathrm{g} / \mathrm{mL}$ & $7(100)$ \\
\hline $5(18.6)$ & PMB and GEN & $1.0 \mu \mathrm{g} / \mathrm{mL}$ & $5(100)$ \\
\hline
\end{tabular}

Note. AMK, amikacin; ATM, aztreonam; CZA, ceftazidime/avibactam; GEN, gentamicin; PMB, polymyxin B.

${ }^{a}$ Antimicrobials tested: amikacin, aztreonam, cefepime, ceftazidime, ceftazidime/avibactam, ciprofloxacin, and piperacillin/tazobactam.

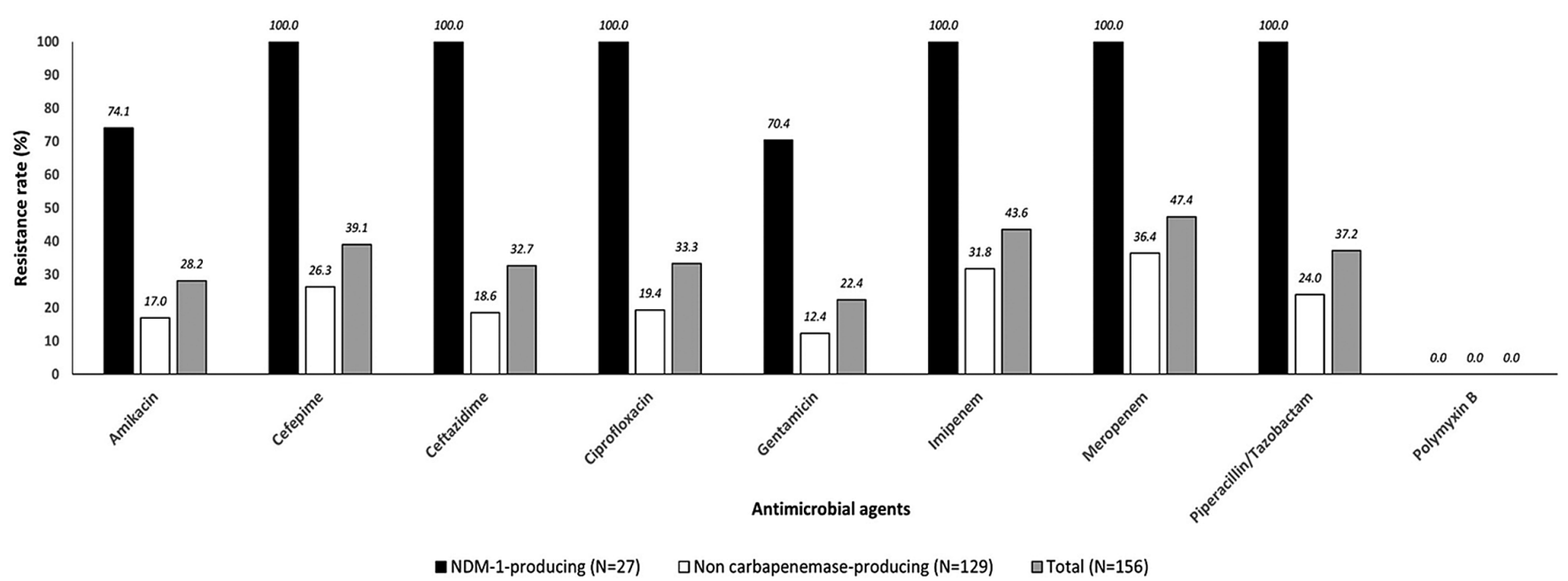

Fig. 1. Antimicrobial resistance rates for the NDM-1-producing and-nonproducing $P$. aeruginosa isolates evaluated in this study.

All NDM-1-producing $P$. aeruginosa isolates showed an in vitro synergism for the ceftazidime/avibactam plus aztreonam. This association has been described as an option to treat infections due to MBL-producing organisms. ${ }^{8,9}$ The rationale is based on the ability of avibactam to inhibit serine- $\beta$ lactamases and the lack of hydrolytic activity of MBLs against aztreonam. ${ }^{10}$ Despite the lack of knowledge about its effectiveness in MBLrelated infections, this synergistic activity may be an important strategy because polymyxins can appear active in vitro but can be flawed in achieving a clinical response.

In conclusion, the emergence of NDM-1-producing $P$. aeruginosa isolates and its impact on a high resistance rate was observed. Because nosocomial antimicrobial resistance can result in higher cost of treatment and increased mortality, it becomes crucial to limit the risk of NDM-1-producing $P$. aeruginosa infection and its spreading. For this, proper use of antimicrobials and strict infection control measures avoiding coinfections in a pandemic scenario is mandatory.

Acknowledgments. The authors thank Pfizer for providing the ceftazidime/ avibactam disks.

Financial support. No financial support was provided relevant to this article.

Conflicts of interest. All authors report no conflicts of interest relevant to this article.

\section{References}

1. Poole K. Pseudomonas aeruginosa: resistance to the max. Front Microbiol 2011;2:65.
2. Livermore D. Interplay of impermeability and chromosomal $\beta$-lactamase activity in imipenem-resistant Pseudomonas aeruginosa. Antimicrob Agents Chemother 1992;36:2046-2048.

3. da Cunha RSR, Carniel E, Narvaez GA, Dias CG, Perez LRR. Impact of the bluecarba rapid test for carbapenemase detection on turnaround time for an early therapy against Pseudomonas aeruginosa. Am J Infect Control 2021;49:352-354.

4. Galetti R, Andrade LN, Varani AM, Darini ALC. SPM-1-producing Pseudomonas aeruginosa ST277 carries a chromosomal pack of acquired resistance genes: an example of high-risk clone associated with 'intrinsic resistome'. J Glob Antimicrob Resist 2019;16:183-186.

5. Linciano P, Cendron L, Gianquinto E, Spyrakis F, Tondi D. Ten Years with New Delhi metallo-beta-lactamase-1 (NDM-1): from structural insights to inhibitor design. ACS Infect Dis 2019;5:9-34.

6. Clinical and Laboratory Standards Institute. Performance Standards for Antimicrobial Susceptibility Testing, 31st edition. Supplement M100. Pittsburgh, PA: CLSI; 2021.

7. Gaudereto JJ, Perdigão Neto LV, Leite GC, et al. Synergistic effect of ceftazidime-avibactam with meropenem against panresistant, carbapenemaseharboring Acinetobacter baumannii and Serratia marcescens investigated using time-kill and disk approximation assays. Antimicrob Agents Chemother 2019;63:e2367-18.

8. Khan A, Shropshire WC, Hanson B, et al. Simultaneous infection with Enterobacteriaceae and Pseudomonas aeruginosa harboring multiple carbapenemases in a returning traveler colonized with Candida auris. Antimicrob Agents Chemother 2020;64:e01466-19.

9. Perez F, El Chakhtoura NG, Papp-Wallace KM, Wilson BM, Bonomo RA. Treatment options for infections caused by carbapenem-resistant Enterobacteriaceae: can we apply "precision medicine" to antimicrobial chemotherapy? Expert Opin Pharmacother 2016;17:761-781.

10. Marshall S, Hujer AM, Rojas LJ, et al. Can ceftazidime-avibactam and aztreonam overcome $\beta$-lactam resistance conferred by metallo- $\beta$-lactamases in Enterobacteriaceae? Antimicrob Agents Chemother 2017;61: e02243-16. 